\title{
Mitochondrial mutational spectrum in poikilothermic versus homeothermic vertebrates: effects of the temperature
}

\author{
Alina G. Mikhaylova \\ Center for Mitochondrial Functional \\ Genomics, Immanuel Kant Baltic \\ Federal University \\ Kaliningrad, Russian Federation \\ The Vavilov Institute of General \\ Genetics RAS, Moscow, Russian \\ Federation \\ polarsong4@gmail.com \\ Victor Shamanskiy \\ Center for Mitochondrial Functional \\ Genomics ,Immanuel Kant Baltic \\ Federal University \\ Kaliningrad, Russian Federation \\ v.a.shamanskiy@gmail.com
}

\author{
Alina A. Mikhaylova \\ Center for Mitochondrial Functional \\ Genomics, Immanuel Kant Baltic \\ Federal University \\ Kaliningrad, Russian Federation \\ mihailovaalina777@yandex.ru \\ Konstantin Gunbin \\ The Institute of Cytology and Genetics \\ of the SB RAS \\ Novosibirsk, Russian Federation \\ genkvg@gmail.com \\ Vsevolod Makeev \\ The Vavilov Institute of General \\ Genetics RAS, Moscow, Russian \\ Federation \\ vsevolod.makeev@gmail.com
}

\author{
Kristina Ushakova \\ ITMO University \\ Saint Petersburg, Russian Federation \\ kristina.ushakova@outlook.com \\ Konstantin Popadin \\ School of Life Sciences \\ Ecole Polytechnique Federale de \\ Lausanne \\ Lausanne, Switzerland \\ Center for Mitochondrial Functional \\ Genomics, Immanuel Kant Baltic \\ Federal University, Kaliningrad, \\ Russian Federation \\ konstantinpopadin@gmail.com
}

\begin{abstract}
Mutational processes of mitochondrial genome in Vertebrates remain unclear. In order to understand them better we obtained mutational spectra, which contain information about single nucleotide substitutions occurred in neutral within-species polymorphisms, uncovering key mutagens of mitochondrial DNA. Here, we analyzed mutational spectra of Actinopterygii and hibernating mammalian species and observed that frequencies of $A>G$ substitutions are sensitive to temperature.
\end{abstract}

Key words - Mutational spectrum, mitochondrial DNA, somatic mutations, Actinopterygii, hibernating mammals

\section{Introduction}

The mutation process is one of the key drivers of evolution. By understanding the rules of mutagenesis, we better learn what distribution of mutations selection we can work with. More and more is found out about the factors affecting the mutagenesis of the nuclear genome: there are many discovered specific mutational signatures of various exogenous (UV light, tobacco smoke ...) and endogenous (DNA polymerase errors, repair enzyme activity) factors. However, for the mitochondrial genome (mtDNA), progress is relatively slow: the main factors (exogenous and endogenous) that determine the specific rules of mtDNA mutagenesis are still unknown. The majority of strong mutagens well known for the nuclear genome (for example, UV-light) do not act the same for mtDNA, which makes mtDNA mutagenesis unique and extremely important, because it is associated with an understanding of selection process of different animal species and largely determines the rate of appearance of human somatic mutations. The global goal of our project is to understand the rules of mtDNA mutagenesis in vertebrates and humans.

\section{RECONSTRUCTION OF MUTATIONAL SPECTRA IN VERTEBRATE SPECIES}

Following the idea that somatic variants in mtDNA are informative to trace different cellular lineages in our body [3] we hypothesized that mtDNA mutational spectrum, defined as a relative frequencies of mtDNA single- nucleotide substitutions, may be used not only for cellular tracing, but may contain information about cellular features and specific functional signatures of a given cell.

In our recent work [1] we firstly analyzed somatic mtDNA mutations from human cancers and healthy human tissues and then focusing on mammalian species we analyzed polymorphic synonymous substitutions in fourfold degenerate sites and found out that mtDNA mutational spectrum indeed is sensitive to several metabolic and ecological properties on all levels (cellular, tissue and organismal). We observed one universal trend: ratio of A to $\mathrm{G}$ transitions $(\mathrm{A}>\mathrm{G}$, heavy chain notation) positively correlates with cellular and organismal longevity. Focusing on mammals, we observed that the fraction of $A>G$ is higher in long- versus short-lived species. This led us to propose that deamination of adenine (the main cause of $A>G$ transitions) is more intense in long-lived (aged) oocytes, typical for long-lived species.

\section{Results}

Here, we extend our analyses towards cold-blooded vertebrates, because substitution frequencies can be affected also by body parameters such as temperature [6], while first dataset represented only uniform species regarding temperature. Analyzing mtDNA mutational spectrum of up to one hundred Actinopterygii species $(\mathrm{N}=128)$ with known longevity (mean maturation time, Tm) and median temperature of environment [5] we observed that the frequency of $A>G$ transitions positively correlates with temperature (results are robust to phylogenetic inertia), while the effect of longevity is only marginally significant. (Fig. 1) Taking into account the fact that observed substitution frequencies can shape specific nucleotide content in neutral positions we analyzed additionally synonymous nucleotide frequencies in more than three hundred $(\mathrm{N}=302)$ whole mitochondrial genomes of Actinopterygii species. As expected we observed decrease of A fraction with increasing temperature, which can be long-term result of $A>G$ substitutions (Fig. 2). 

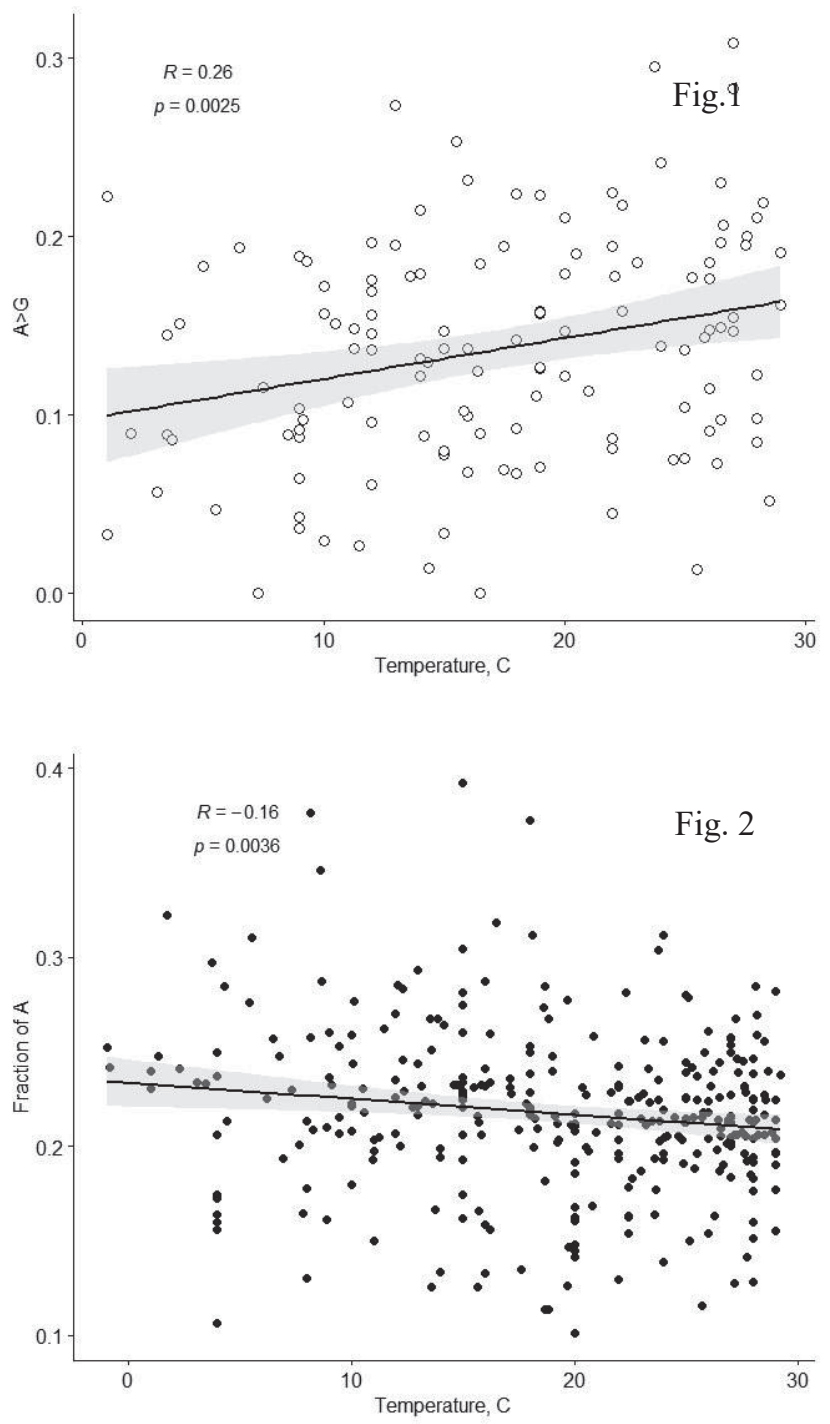

Altogether, we conclude that $A>G$ is sensitive to both temperature and lifespan, with temperature predominantly shaping variation in mtDNA mutational spectrum of poikilotherms, while longevity is the main factor in homeotherms. To prove this, we compared hibernating mammalian species (HibDay in fig. 3), Monotremata species and Marsupials species (MonoMarsHG in fig. 3) with nonhibernating placental ones (Warm in fig. 3) $(\mathrm{N}=350)$, controlling for generation length $[2,4]$. We observed a increased fraction of $\mathrm{A}$ in hibernating, Monotremata, Marsupial species, in line with their expected decreased temperature.

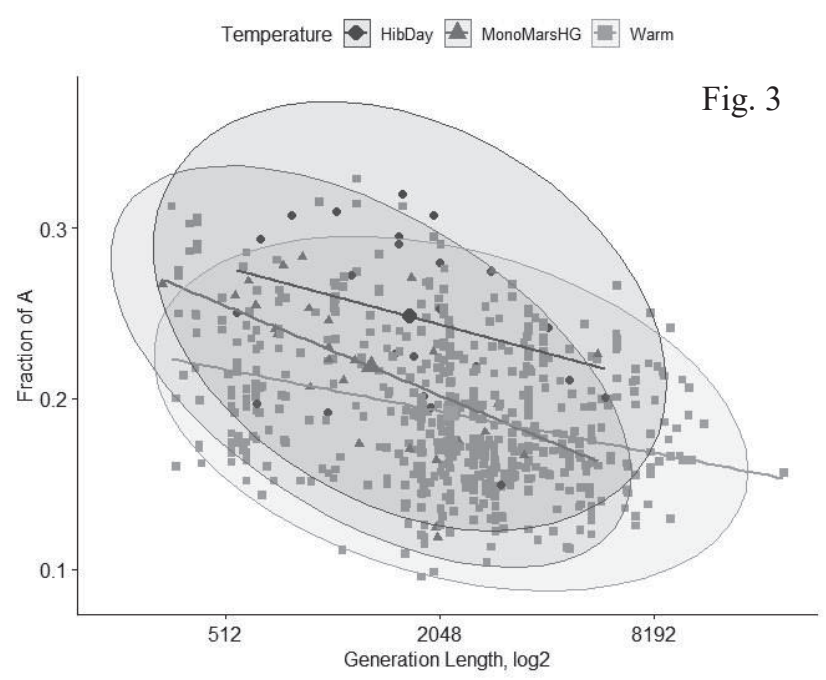

Multiple models were performed for all analyses to improve results. Taking into account that $A>G$ is one of the most common transitions in mtDNA we conclude that the whole mtDNA mutational spectrum is associated with temperature and longevity. This new information should be taken into account in numerous ecological, population and evolutionary projects where mitochondrial genes are the most frequently used markers.

\section{Acknowledgment}

This work is supported by the 5 Top 100 Russian Academic

Excellence Project at the Immanuel Kant Baltic Federal University, by the Russian Foundation for Basic Research grant 18-29-13055.

\section{References}

[1] A. G. Mikhaylova, A. A. Mikhailova, K. Ushakova, E. Tretiakov, A. Yurchenko, M. Zazhytska, D.A. Knorre, E. Zdobnov, Z. Fleischmann, S. Annis, M. Franco, K. Wasko, W.S. Kunz, I. Mazunin, S. Nikolaev, A. Reymond, K. Khrapko, K. Gunbin, View ORCID ProfileK. Popadin, Mitochondrial mutational spectrum provides an universal marker of cellular and organismal longevity", bioRxiv 589168; doi: https://doi.org/10.1101/589168.

[2] J. P. de Magalhaes and J. Costa, "A database of vertebrate longevity records and their relation to other life-history traits.", 2009, Journal of Evolutionary Biology 22(8):1770-1774.

[3] L. S. Ludwig, C. A. Lareau, J. C. Ulirsch, E. Christian, C. Muus, L.

[4] H. Li, K. Pelka, et al. 2019. "Lineage Tracing in Humans Enabled by Mitochondrial Mutations and Single-Cell Genomics." Cell 176 (6): 1325-39.e22.

[5] M. Pacifici, L. Santini, M. Di Marco, L. Santini, D. Baisero, L. Francucci, G. Grottolo Marasini, P. Visconti, C. Rondinini, Generation length for mammals. 2013, Nature Conservation 5: 89-94.

[6] R. Froese and D. Pauly. Editors. 2019. FishBase. World Wide Web electronic publication. www.fishbase.org, (12/2019)

[7] W. Zheng, K. Khrapko, H. A. Coller, W. G. Thilly, W. C. Copeland Origins of human mitochondrial point mutations as DNA polymerase gamma-mediated errors. Mutat Res. 2006 Jul 25;599(1-2):11-20. Epub 2006 Feb 20. 